\title{
Puntaje predictivo de resultado en la cirugía reconstructiva de miembros inferiores. Ensayo preliminar
}

\author{
Mauro R. Vivas \\ Unidad de Miembro Inferior, Servicio de Ortopedia y Traumatología, Hospital de Alta Complejidad en Red "El Cruce”, \\ Dr. Néstor C. Kirchner, Florencio Varela, Buenos Aires, Argentina
}

\begin{abstract}
RESUMEN
Introducción: Las secuelas postraumáticas en los miembros son un cuadro de muy difícil abordaje por la gravedad de las lesiones, la falta de protocolos de tratamiento y especialmente por la elección de la mejor alternativa. El objetivo de este estudio fue analizar el resultado del tratamiento reconstructivo con un puntaje prequirúrgico diseñado para tal fin y evaluar su predictibilidad de resultado. Materiales y Métodos: Se realizó un análisis retrospectivo de tipo cohorte de los resultados del tratamiento con fijación externa en 32 pacientes con secuela traumática de la pierna en los últimos siete años que fueron estadificados con un puntaje preoperatorio elaborado para tal fin y comparado con el resultado obtenido con la tabla de resultados de la ASAMI con dos años de seguimiento mínimo. Resultados: Se obtuvo una relación directa entre la estadificación prequirúrgica y el resultado final. Dicha relación se ve reflejada en el alto porcentaje de excelentes resultados en pacientes de bajo riesgo (50\%) y una tasa del $60 \%$ de malos resultados en pacientes de alto riesgo. Conclusiones: La evidencia actual es contradictoria y se discute cuál es la mejor opción de tratamiento. Los pacientes considerados de alto riesgo tienen un alto porcentaje de malos resultados y tal vez no se beneficien con la cirugía reconstructiva.
\end{abstract}

Palabras clave: Tibia; Ilizarov; secuela postraumática.

Nivel de Evidencia: IV

Predictive Outcome Score in Lower Limb Reconstructive Surgery. Preliminary Test

\section{ABSTRACT}

Introduction: Post-traumatic sequelae in the limbs are a very difficult condition to approach due to the severity of the injuries and the lack of treatment protocols, which considerably hinder the choice of the best treatment alternative. The objective of this study was to analyze the outcomes of reconstructive treatment with a pre-surgical score designed ad-hoc and to evaluate its predictability. Materials and Methods: A retrospective cohort-type analysis of the outcomes of external fixation treatment was performed in 32 patients with traumatic leg sequelae in the last 7 years who were staged with a pre-operative score designed ad-hoc. This was compared with the results obtained with the ASAMI score with a minimum 2-year follow-up. Results: We obtained a direct relationship between pre-surgical staging and the final outcome. This relationship is reflected by obtaining a high percentage of excellent outcomes in low-risk patients $(50 \%)$ and $60 \%$ of poor outcomes in high-risk patients. Conclusions: The current evidence is contradictory and there is controversy over which is the best treatment option. We found that high-risk patients have a high percentage of poor outcomes and may not benefit from reconstructive surgery.

Key words: Tibia; llizarov; post-traumatic sequelae.

Level of Evidence: IV

\section{INTRODUCCIÓN}

Las secuelas postraumáticas de los miembros se han convertido en un cuadro endémico en nuestro país. La Organización Panamericana de la Salud informó, en 2014, que se habían producido 15.000 lesiones permanentes anuales por accidentes de tránsito, en la Argentina. ${ }^{1}$ Las publicaciones sobre alternativas terapéuticas para las secuelas postraumáticas son muy variadas y, en su mayoría, tienen un bajo nivel de evidencia (reportes y series de casos).

Recibido el 21-6-2020. Aceptado luego de la evaluación el 15-8-2020 • Dr. MAURO R. VIVAS • vivasmauro@ hotmail.com

Cómo citar este artículo: Vivas MR. Puntaje predictivo de resultado en la cirugía reconstructiva de miembros inferiores. Ensayo preliminar. Rev Asoc Argent Ortop Traumatol 2021;86(6):771-

779. https://doi.org/10.15417/issn. 1852-7434.2021.86.6.1140 
Numerosas publicaciones sobre lesiones graves en pacientes agudos evalúan las indicaciones de amputación o reconstrucción. Se han diseñado varios puntajes para la toma de decisiones en pacientes con un trauma agudo, con sus fortalezas y debilidades, que sirven de guía para este proceso. Los ítems más utilizados en estos puntajes son el estado hemodinámico, el tiempo de isquemia, el daño óseo y el muscular. Sin embargo, aún no se ha llegado a un consenso y no tienen validez en las secuelas postraumáticas. ${ }^{2,3}$ La Limb Lengthening Reconstruction Society (LLRS) diseñó el LLRS AIM Index para evaluar el nivel de complejidad del cuadro por tratar; sin embargo, no ha ganado popularidad con los años y su validez no ha sido evaluada por completo. ${ }^{4}$ Tampoco se lo ha relacionado con los resultados al final del tratamiento.

La cirugía reconstructiva de los miembros es, en muchos casos, un gran desafío en cuanto a la magnitud del daño, sus comorbilidades y el método que se aplique para dicho fin. La fijación externa como método para la recuperación de stock óseo en pacientes con antecedentes infecciosos o lesiones tegumentarias no apropiadas para la osteosíntesis interna es la alternativa más utilizada en estas circunstancias. ${ }^{5}$

El objetivo de este estudio fue evaluar, de manera retrospectiva, el diseño de un puntaje de riesgo prequirúrgico para secuelas postraumáticas de la pierna con respecto a los resultados obtenidos con la clasificación de resultados de la ASAMI y, de esta manera, obtener información que nos permita decidir, de forma más objetiva, en los futuros casos y poder responder la siguiente pregunta: ¿la cirugía de rescate con fijación externa es válida en pacientes de alto riesgo?

\section{MATERIALES Y MÉTODOS}

Se llevó a cabo un análisis retrospectivo de pacientes $>17$ años que tenían secuelas postraumáticas en la pierna con defectos óseos no articulares tratados mediante fijación externa en dos instituciones, por el mismo equipo quirúrgico, durante los últimos siete años, con un seguimiento mínimo de dos años. Se excluyó a los pacientes tratados con un cuadro agudo ( $<3$ meses del trauma) y a aquellos con desejes y discrepancia de los miembros inferiores no asociados a lesiones traumáticas. Se decidió incluir solo las lesiones del segmento de la pierna y las tratadas con fijación externa para formar una población homogénea y disminuir factores que influyan en el resultado final. Para este estudio, se diseñó un puntaje preoperatorio (Tabla 1) que evalúa los principales problemas que enfrenta el cirujano en el tratamiento de esta patología, cuyo fin es obtener un miembro funcional y sin dolor durante la marcha. Los problemas incluidos como determinantes de resultado fueron: el compromiso de partes blandas, el estado infectológico, el tipo y la magnitud del defecto óseo, el daño articular y las comorbilidades generales del paciente (edad >60 años, artrosis o rigidez articular, segmento multioperado, etc.).

Durante el diseño de este cuestionario de valoración clínica o puntaje, se propuso cubrir todas las características de los diseños de mayor calidad, como la validez de contenido, de criterio, de constructo, consistencia interna, efecto suelo y techo y fidelidad prueba-prueba.

A cada ítem se le otorgó un valor numérico basado en la incidencia de riesgo descrita en otras publicaciones ${ }^{6-8}$ y en la experiencia del equipo quirúrgico. Estos valores se sumaron al final de la evaluación del paciente y posteriormente, se definieron valores de corte para clasificar a los pacientes por tipo de riesgo, donde un valor inferior a 16 se considera de bajo riesgo, entre 16 y 30, de mediano riesgo y $>30$ de alto riesgo.

La valoración radiográfica se realizó mediante telerradiografías impresas (radiografías tomadas a 3,05 m de distancia, compensando la discrepancia para lograr nivelar la pelvis y las rótulas al frente) y sobre la medición digital con el programa IntelliSpace Pacs de Phillips. La descripción de la calidad de las partes blandas, el estado infectológico, el compromiso articular, las comorbilidades, etc. se obtuvieron del interrogatorio y el examen físico en las primeras consultas.

Finalmente, una vez concluido el tratamiento y tras dos años de seguimiento mínimo, se evaluó el resultado final con el puntaje de resultados de la ASAMI (Tabla 2).

\section{RESULTADOS}

La búsqueda arrojó un total de 32 pacientes: 25 hombres y siete mujeres con una edad promedio de 38.3 años (rango 19-63). Todos tenían una discrepancia $\geq 1 \mathrm{~cm}$, desejes o antecedentes infecciosos que justificaban la fijación externa como la opción más adecuada. Se excluyó a los pacientes que fueron tratados con otros medios y a tres por no cumplir con los criterios de seguimiento a largo plazo. En la Tabla 3, se detallan las características de la muestra.

Los resultados generales fueron incluidos en una tabla con el puntaje correspondiente al obtenido en el preoperatorio (Tabla 4), y los resultados finales globales se detallan en la Figura 1. 
Tabla 1. Puntaje de evaluación*

\begin{tabular}{|c|c|c|}
\hline Factores & Compromiso & Puntaje \\
\hline \multirow[t]{5}{*}{ Partes blandas } & Cobertura normal & 0 \\
\hline & Colgajo vascularizado & 3 \\
\hline & Colgajo libre & 6 \\
\hline & Tejido de granulación & 8 \\
\hline & Defecto de cobertura & 10 \\
\hline \multirow[t]{5}{*}{ Infección } & Negativa & 0 \\
\hline & Antecedente de infección & 3 \\
\hline & Positiva a germen sensible a antibióticos & 6 \\
\hline & Positiva a dos o más gérmenes & 10 \\
\hline & Positiva a germen resistente & 15 \\
\hline \multirow[t]{5}{*}{ Defecto óseo } & Sin defecto óseo & 0 \\
\hline & $<10 \%$ & 3 \\
\hline & $11-20 \%$ & 6 \\
\hline & $20-30 \%$ & 10 \\
\hline & $>30 \%$ & 15 \\
\hline \multirow[t]{4}{*}{ Compromiso articular } & Sin compromiso articular evidente & 0 \\
\hline & Rigidez /contractura & 5 \\
\hline & Lesión de nervio ciático poplíteo externo & 8 \\
\hline & Alteración mecánica (ósea o capsuloligamentaria) & 10 \\
\hline \multirow[t]{8}{*}{ Comorbilidades } & Sin comorbilidades & 0 \\
\hline & Tabaquista ( 5 moderado; 10 severo) & \\
\hline & Índice de masa corporal $>30$ & 5 \\
\hline & Artrosis postraumática & 5 \\
\hline & Edad $>60$ años & 5 \\
\hline & Diabetes $>10$ años & 10 \\
\hline & Múltiples operaciones previas (más de 5) & 5 \\
\hline & Compromiso vascular o nervioso & 10 \\
\hline Total & & 85 \\
\hline
\end{tabular}

Se detallan los elementos considerados como determinantes para el resultado final. 
Tabla 2. Puntaje de resultados de la ASAMI

\begin{tabular}{|c|c|c|c|c|c|}
\hline Variable ósea & & & & & \\
\hline & Excelente & Bueno & Bueno & Regular & Malo \\
\hline Consolidación sin infección & Sí & Sí & Sí & Sí & No \\
\hline Deformidad residual & $<7^{\circ} \mathrm{mm}$ & $<7^{\circ} \mathrm{mm}$ & $>7^{\circ} \mathrm{mm}$ & $>7^{\circ} \mathrm{mm}$ & \\
\hline Discrepancia de longitud & $<2,5 \mathrm{~cm}$ & $>2,5 \mathrm{~cm}$ & $<2,5 \mathrm{~cm}$ & $>2,5 \mathrm{~cm}$ & \\
\hline Variable funcional & & & & & \\
\hline & Debilidad & Rigidez en equino & Distrofia de partes blandas & Dolor & Inactividad laboral \\
\hline Excelente & No & No & No & No & No \\
\hline Bueno & 2 de 5 & variables & & & \\
\hline Regular & 3 o 4 de 5 & variables & & & \\
\hline Malo & Sí & Sí & Sí & Sí & Sí \\
\hline
\end{tabular}

Tabla 3. Características de los pacientes $(n=31)$

\begin{tabular}{|l|c|}
\hline Edad y media & 37 años $(61-19)$ \\
\hline Hombre:Mujer & $14: 7$ \\
\hline Derecho:izquierdo & $9: 12$ \\
\hline Defecto óseo: media e intervalo & $4,9 \mathrm{~cm}(0,5-14)$ \\
\hline Signos de infección activa/negativa & $14 / 22$ \\
\hline Defectos de cobertura & $5 / 22$ \\
\hline Demora hasta el tratamiento & 16.6 meses $(5-32)$ \\
\hline
\end{tabular}


Tabla 4. Resultados pretratamiento y postratamiento (seguimiento mínimo de 2 años)

\begin{tabular}{|c|c|c|c|c|c|c|c|c|c|}
\hline Paciente & Edad & Segmento & Sexo & $\begin{array}{l}\text { Puntaje de } \\
\text { riesgo }\end{array}$ & $\begin{array}{l}\text { Resultado } \\
\text { final }\end{array}$ & Lado & $\begin{array}{c}\text { Infección } \\
\text { activa }\end{array}$ & $\begin{array}{c}\text { Defecto } \\
\text { óseo }\end{array}$ & $\begin{array}{l}\text { Demora } \\
\text { (meses) }\end{array}$ \\
\hline 1 & 29 & Pierna & M & 49 & Bueno & I & Sí & 14 & 5 \\
\hline 2 & 29 & Pierna & M & 8 & Excelente & I & No & 7 & 5 \\
\hline 3 & 45 & Pierna & M & 26 & Bueno & I & Sí & 8 & 10 \\
\hline 4 & 25 & Pierna & M & 3 & Excelente & D & No & 3 & 23 \\
\hline 5 & 41 & Pierna & $\mathrm{F}$ & 44 & Malo & I & Sí & 10 & 6 \\
\hline 6 & 33 & Pierna & M & 30 & Excelente & I & Sí & 6 & 18 \\
\hline 7 & 37 & Pierna & M & 25 & Excelente & I & No & 8 & 13 \\
\hline 8 & 42 & Pierna & M & 16 & Regular & I & Sí & 6 & 43 \\
\hline 9 & 52 & Pierna & M & 24 & Bueno & D & No & 2 & 16 \\
\hline 10 & 53 & Pierna & $\mathrm{F}$ & 29 & Excelente & D & Sí & 2 & 21 \\
\hline 11 & 42 & Pierna & M & 11 & Excelente & I & No & 3 & 35 \\
\hline 12 & 44 & Pierna & M & 26 & Bueno & I & Sí & 6 & 19 \\
\hline 13 & 34 & Pierna & M & 8 & Excelente & D & No & 5 & 13 \\
\hline 14 & 46 & Pierna & M & 8 & Bueno & I & No & 2 & 9 \\
\hline 15 & 30 & Pierna & M & 17 & Excelente & I & No & 3 & 23 \\
\hline 16 & 48 & Pierna & $\mathrm{F}$ & 6 & Excelente & D & No & 3 & 14 \\
\hline 17 & 39 & Pierna & M & 34 & Malo & D & Sí & 2 & 27 \\
\hline 18 & 53 & Pierna & M & 57 & Regular & & Sí & 7 & 4 \\
\hline 19 & 27 & Pierna & M & 17 & Bueno & I & No & 4 & 11 \\
\hline 20 & 48 & Pierna & M & 14 & Bueno & I & No & 4 & 25 \\
\hline 21 & 19 & Pierna & $\mathrm{F}$ & 21 & Regular & I & Sí & 11 & 14 \\
\hline 22 & 24 & Pierna & M & 29 & Excelente & I & Sí & 1 & 5 \\
\hline 23 & 19 & Pierna & $\mathrm{F}$ & 17 & Regular & I & No & 5 & 7 \\
\hline 24 & 27 & Pierna & M & 16 & Bueno & D & No & 5 & 27 \\
\hline 25 & 29 & Pierna & M & 6 & Excelente & I & No & 1 & 14 \\
\hline 26 & 63 & Pierna & $\mathrm{F}$ & 27 & Bueno & D & Sí & 4 & 32 \\
\hline 27 & 42 & Pierna & M & 6 & Excelente & I & No & 0 & 13 \\
\hline 28 & 23 & Pierna & M & 3 & Excelente & D & No & 0 & 9 \\
\hline 29 & 30 & Pierna & M & 50 & Bueno & I & Sí & 11 & 7 \\
\hline 30 & 46 & Pierna & M & 30 & Bueno & I & No & 6 & 28 \\
\hline 31 & 54 & Pierna & $\mathrm{F}$ & 61 & Malo & I & Sí & 4 & 20 \\
\hline \multirow[t]{2}{*}{32} & 53 & Pierna & M & 21 & Excelente & I & No & 3 & 9 \\
\hline & 38,313 & & & 23,09 & & & & 4,88 & 16.41 \\
\hline
\end{tabular}

$\mathrm{M}=$ masculino, $\mathrm{F}=$ femenino, $\mathrm{I}=$ izquierdo, $\mathrm{D}=$ derecho 


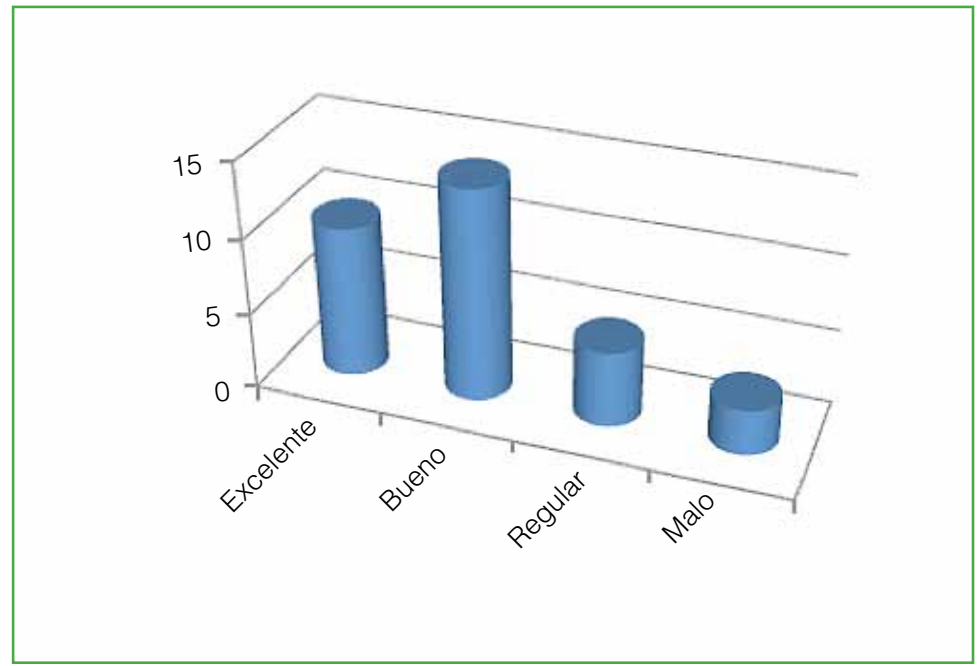

Figura 1. Resultados globales postratamiento $(\mathrm{n}=32)$.

Después se cruzaron los datos para observar la relación entre el resultado obtenido de la evaluación preoperatoria y el resultado final del paciente (Tabla 5, Figura 2).

Tabla 5. Resultados versus riesgo

\begin{tabular}{|l|c|c|c|c|}
\hline Riesgo de complicaciones & Excelente & Bueno & Regular & Malo \\
\hline Bajo & 8 & 2 & 0 & 0 \\
\hline Medio & 6 & 7 & 3 & 0 \\
\hline Alto & 0 & 1 & 2 & 3 \\
\hline Total & 14 & 10 & 5 & 3 \\
\hline
\end{tabular}

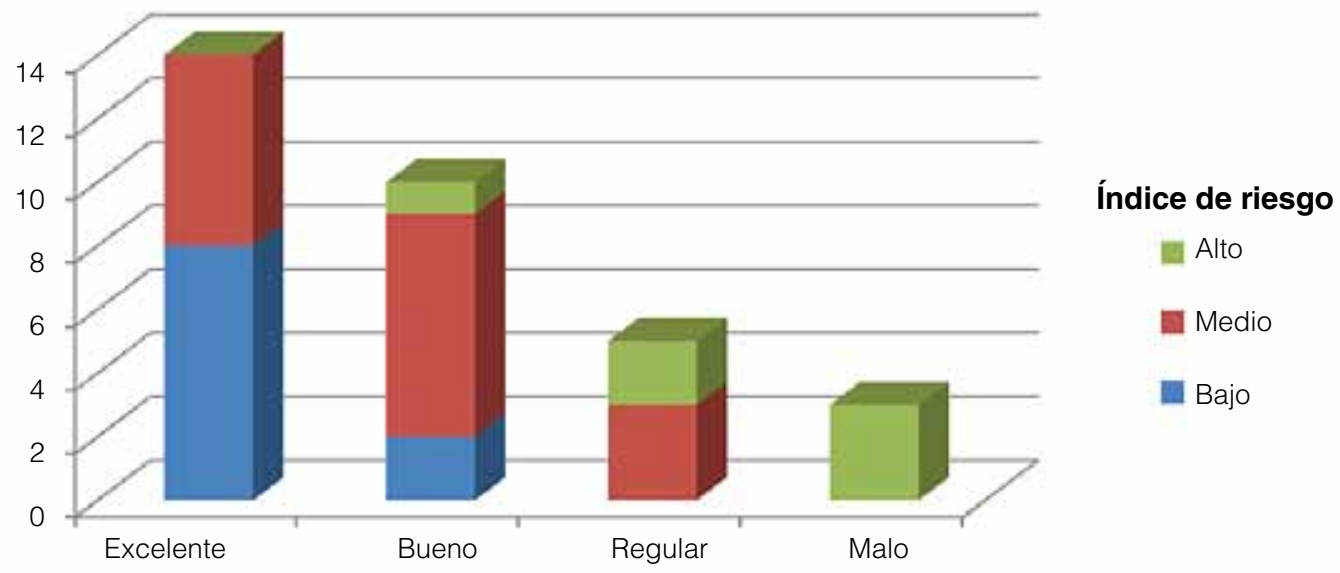

Resultado funcional y radiográfico según el puntaje de la ASAMI

Figura 2. Relación entre el riesgo del paciente y el resultado final $(n=31)$. Se observó una relación directa entre el riesgo y el resultado final. 
Los resultados obtenidos muestran una relación directa entre la tasa de riesgo analizado en la evaluación preoperatoria y el valor de resultado final. Dicha relación se ve reflejada en el alto porcentaje de excelentes resultados en pacientes de bajo riesgo (60\%), mientras que, en pacientes de alto riesgo, la tasa de malos resultados llegó al 60\%.

Todos los pacientes tuvieron una buena adherencia al tratamiento y una familia continente por lo que no se tuvieron en cuenta en el análisis de resultado. Tres de 32 pacientes obtuvieron un mal resultado $(9,3 \%)$, todos de alto riesgo (60\% del total).

\section{DISCUSIÓN}

En la bibliografía actual, no hemos encontrado evidencia de un protocolo prequirúrgico de características similares que analice los factores que influyen en el resultado final del tratamiento de secuelas postraumáticas de los miembros. El mayor porcentaje de publicaciones sobre reconstrucción ósea en lesiones graves de los miembros es de nivel 4 (reporte o series de casos).

La decisión de reconstrucción ósea o de amputación en secuelas postraumáticas es muy subjetiva hoy en día, está basada en la experiencia del cirujano debido a que la evidencia científica no es contundente al respecto. Esto se debe a que no existen puntajes o algoritmos de tratamiento para esta patología secuelar. Tres publicaciones son el producto del estudio de cohorte Lower Extremity Assessment Project (LEAP), donde se evaluó el impacto de la enfermedad en la calidad de vida de pacientes amputados y aquellos que se sometieron a una reconstrucción ósea, un estudio que incluyó 613 casos. El primero de ellos, publicado por Bosse y cols., en 2002, no encontró diferencias estadísticamente significativas en los dominios de las funciones o en el aspecto psicológico. ${ }^{9}$ La segunda publicación fue de la Mackenzie y cols., en 2005, quienes revisaron nuevamente a los pacientes con un mínimo de siete años de seguimiento y, pese a no hallar diferencias estadísticamente significativas, detectaron un deterioro funcional y psicológico con ambas opciones de tratamiento. ${ }^{10}$ Los factores determinados como marcadores de riesgos para este descenso fueron: el tabaquismo, la pobreza, el estado de salud previo y la falta de contención familiar. ${ }^{11}$ Por último, en el tercer informe, Harris y cols. evaluaron las complicaciones de los pacientes de la cohorte LEAP con un seguimiento mínimo de dos años y destacaron como relevante que la infección del sitio operatorio fue la más frecuente en los amputados y que la tasa de falta de consolidación fue del 31,5\% en pacientes que se sometieron al rescate de miembros. Los autores concluyen en que si bien en los primeros seis meses, los pacientes más críticos son los sometidos a una amputación, quienes fueron sometidos a una reconstrucción ósea deben esperar una tasa de complicaciones alta. ${ }^{6}$

Desde otro punto de vista, algunos reportes de especialistas en rehabilitación concluyen en que, a pesar de mejorar un 30\% la calidad de vida luego de la amputación, los resultados finales siguen siendo pobres. ${ }^{12,13}$

Otro punto importante por tener en cuenta para la toma de decisiones es la magnitud del daño por reparar y el deterioro de dicho miembro. En la gran mayoría de los informes, no está determinada la cantidad máxima del alargamiento o transporte, pero sí hay publicaciones que afirman que alargar más de un 20-25\% de la longitud total del segmento afectado en un solo evento puede llevar a una mayor incidencia de complicaciones. La duración total de la fijación externa en elongaciones óseas femorales oscila entre 24 y 90 semanas por evento, con índices tiempo/ longitud promedio de 6.23 semanas por cada centímetro elongado, aunque algunos autores han publicado períodos mucho más prolongados según la magnitud de la osteogénesis. ${ }^{14,15}$ La necesidad de prolongar el uso del dispositivo puede ser considerada un factor de riesgo de más complicaciones.

En 2005, Capomassi y cols. publicaron una serie de 24 casos tipo B según la clasificación de Paley, la tasa de resultados buenos y excelentes fue del 83\%. Los autores concluyeron en que la osteogénesis en distracción es un método vigente para tratar defectos osteocutáneos graves de los miembros. ${ }^{16}$

En 2016, el Ministerio de Salud y Protección Social de Colombia y la Universidad de Antioquia elaboraron una guía de práctica clínica basada en la evidencia sobre la cirugía de reconstrucción comparada con la amputación en pacientes que sufrían lesiones graves de los miembros inferiores. A pesar de no ser contundente por su bajo nivel de evidencia, los autores sugieren la reconstrucción en pacientes con trauma grave $>16$ años con el fin de disminuir los costos y mejorar la función a largo plazo. A su vez, en esa publicación, se evidencian tres datos interesantes que modifican los resultados finales: si la población en estudio es civil o militar, si la amputación se realiza de manera temprana ( $<90$ días) o tardía y si el nivel de amputación es por encima o debajo de la rodilla. Los estudios en personal militar obtuvieron resultados favorables de la amputación, informaron menor estrés postraumático, menos dolor crónico y una tasa de complicaciones más baja. Con respecto al nivel de reconstrucción, el resultado fue mejor cuando el daño comprometía la pierna, mientras que los daños en el fémur causaron una tasa de complicaciones más alta y los resultados con la amputación fueron mejores. ${ }^{17}$ 
Las debilidades de nuestro estudio son el puntaje con valores numéricos parcialmente subjetivos, es decir, la validez de constructo y la validez interna no pudieron ser evaluadas debido a una casuística baja y un seguimiento a mediano y corto plazo. Como fortalezas consideramos que la prueba tiene buena validez de contenido, fiabilidad prueba-prueba e interobservador, así como sensibilidad al cambio y posible validación y traducción.

\section{CONCLUSIONES}

El puntaje predictivo de riesgo es una herramienta útil y tiene como meta dar objetividad en la toma de decisiones futuras, principalmente, cuando la cirugía reconstructiva y la amputación no tienen una indicación clara. Además, el puntaje evidenciará las complicaciones no resueltas al final del tratamiento (sensibilidad al cambio) y se podrán ver, de manera cuantitativa, los valores obtenidos. Por lo tanto y, sobre la base de los resultados de este estudio, los pacientes con un alto riesgo de complicaciones tienen una alta incidencia de resultados funcionales y radiográficos pobres y malos, y no sería recomendable la cirugía reconstructiva con el método analizado.

Conflictos de intereses: El autor no declara conflictos de intereses.

\section{BIBLIOGRAFÍA}

1. Organización Panamericana de la Salud. Traumatismos causados por el tránsito y discapacidad. Disponible en: https://iris.paho.org/handle/10665.2/31080

2. Shanmuganathan R. The utility of scores in the decision to salvage or amputation in severely injured limbs. Indian $J$ Orthop 2008;42(4):368-76. https://doi.org/10.4103/0019-5413.43371

3. Russell WL, Sailors DM, Whittle TB, Fisher DF Jr, Burns RP. Limb salvage versus traumatic amputation. A decision based on a seven-part predictive index. Ann Surg 1991;213:473-81. https://doi.org/10.1097/00000658-199105000-00013

4. McCarthy JJ, Iobst CA, Rozbruch SR, Sabharwal S, Eismann EA. Limb Lengthening and Reconstruction Society AIM index reliably assesses lower limb deformity. Clin Orthop Relat Res 2013;471(2):621-7. https://doi.org/10.1007/s11999-012-2609-8

5. Vivas MR, Cordano OR. Utilización del método Ilizarov para el tratamiento de desejes y discrepancias postraumáticos de la tibia. Serie de casos. Rev Asoc Argent Ortop Traumatol 2019;84(2):136-42. https://doi.org/10.15417/issn.1852-7434.2019.84.2.827

6. Harris AM, Althausen PL, Kellam J, Bosse MJ, Castillo R; Lower Extremity Assessment Project (LEAP) Study Group. Complications following limb-threatening lower extremity trauma. J Orthop Trauma 2009;23(1):1-6. https://doi.org/10.1097/BOT.0b013e31818e43dd

7. Howe H Jr, Poole G, Hansen K, Clark T, Plonk G, Koman L, et al. Salvage of lower extremities following combined orthopedic and vascular trauma. A predictive salvage index. Am Surg 1987;53:205-8. PMID: 3579025

8. Johansen K, Daines M, Howey T, Helfet D, Hansen ST Jr. Objective criteria accurately predict amputation following lower extremity trauma. J Trauma 1990;30:568-73. https://doi.org/10.1097/00005373-199005000-00007

9. Bosse MJ, MacKenzie EJ, Kellam JF, Burgess AR, Webb LX, Swiontkowski MF, et al. An analysis of outcomes of reconstruction or amputation after leg-threatening injuries. N Engl J Med 2002;347(24):1924-31. https://doi.org/10.1056/NEJMoa012604

10. MacKenzie EJ, Bosse MJ, Pollak AN, Webb LX, Swiontkowski MF, Kellam JF, et al. Long-term persistence of disability following severe lower-limb trauma. Results of a seven-year follow-up. J Bone Joint Surg Am 2005;87(8):1801-9. https://doi.org/10.2106/JBJS.E.00032

11. McNamara MG, Heckman JD, Corley EG. Severe open fracture of the lower extremity: a retrospective evaluation of the Mangled Extremity Severity Score. J Orthop Trauma 1994;8:81-7. https://doi.org/10.1097/00005131-199404000-00001

12. Tonon da Luz SC, Avila AOV, Olivera TP, Andrade MC, Ventoza Lacunza C. Valoración del daño corporal en amputados de miembros inferiores: prueba de sensibilidad, postura, sobrecarga articular y calidad de vida. Trauma 2010;21(3):178-83. Disponible en: https://app.mapfre.com/fundacion/html/revistas/trauma/v21n3/pdf/02_08.pdf 
13. Samitier CB, Guirao L, Pleguezuelos E, Perez Mesquida ME. Valoración de la movilidad en pacientes con amputación de miembro inferior. Rehabilitación 2011;45(1):61-6. https://doi.org/10.1016/j.rh.2010.09.006

14. Cirpar M, Cetik O, Uslu M, Eksioglu F. Common complications of segmental bone transport with Ilizarov technique in defective tibia pseudoarthrosis: a review. Eur J Orthop Surg Traumatol 2006;16:380-5. https://doi.org/10.1007/s00590-006-0089-9

15. Kawoosa AA, Majid S, Halwai MA, Mohd Mir MR, Mir GR. Femoral lengthening by Ilizarov technique. Results and complications. JK Science 2004;6(3):163. Disponible en: http://jkscience.org/archive/volume63/femoral.pdf

16. Capomassi A, Oliveto R, D’Elia M, Oliveto A, Lombardo E. Pseudoartrosis con pérdida ósea de fémur y de tibia. Rev Asoc Argent Ortop Traumatol 2005;70(1):45-52. Disponible en: https://aaot.org.ar/wp-content/uploads/2021/10/Rev-Asoc-Argent-Ortop-Traumatol-2005-70-1-45-52_Capomassi.pdf

17. Puerta-Gómez A, Lugo-Agudelo LH, Castaño-González A, Posada-Borrero AM. Reconstrucción versus amputación en pacientes con lesiones graves del miembro inferior. Recomendaciones basadas en la evidencia para la Guía de Práctica Clínica de personas amputadas de miembro inferior en Colombia. Iatreia 2006;29(Suppl 4):S72-S81. Disponible en: http://hdl.handle.net/10495/12633 